\title{
Flora da Reserva Ducke, Amazonas, Brasil: Cyperaceae
}

\author{
David A. Simpson ${ }^{1}$
}

Bruhl, J. J. 1995. Sedge genera of the world: relationships and a new classification of the Cyperaceae. Aust. Syst. Bot. 8: 125-305.

Dahlgren, R. M. T.; Clifford, H. T. \& Yeo, P. F. 1985. The families of Monocotyledons. Springer-Verlag, Berlin and New York. 520p.

Goetghebeur, P. 1998. Cyperaceae. In: K. Kubitzki (ed.). The families and genera of vascular plants. SpringerVerlag, Berlin, Germany. Vol. 4, pp. 141-190.

Linder, H. P. \& Kellogg, E. A. 1995. Phylogenetic patterns in the commelinid clade. In: P. J. Rudall; P. J. Cribb; D. F. Cutler \& C. J. Humphries (eds.). Monocotyledons: systematics and evolution. Royal Botanic Gardens, Kew. Pp. 473-496.

Simpson, D. A. 1995. Relationships within Cyperales. In: P. J. Rudall; P. J. Cribb; D. F. Cutler \& C. J. Humphries (eds.): Monocotyledons: systematics and evolution. Royal Botanic Gardens, Kew. Pp. 497-509

Annual or perennial, rhizomatous to stoloniferous herbs. Stems (culms) simple, often 3 -sided. Leaves basal and/or cauline, often 3-ranked, comprising blade and sheath, sometimes sheath only present; blade usually linear, grass-like, sometimes broader and constricted into a pseudopetiole below; sheath open or closed; ligule often present, sometimes on opposite side to blade. Involucral bracts 1several, leaf-like or glume-like. Inflorescence unbranched to simply, compoundly or decompoundly branched and umbel-like, or paniculate, comprising 1-many ultimate inflorescence units (spikelets or spicoids). Spikelets comprising 1-many glumes, the glumes membranous to coriaceous, spirally arranged or 2-ranked, each subtending a single bisexual or unisexual flower or sterile, the spikelet sometimes reduced to a single flower and aggregated into spikes; spicoids (tribe Hypolytreae only) comprising a terminal female flower, 2-12 membranous scale-like floral bracts on a much reduced axis, the lowest 2 bracts opposite and keeled, some of the bracts subtending a male flower, the spicoid subtended and usually hidden by a glume-like spicoid bract, these spirally arranged and aggregated into spikelet-like spikes. Perianth absent or reduced to bristles or scale-like segments. Stamens 1-3; anthers basifixed. Stigmas 2-3, rarely style undivided, the base sometimes persistent and variously shaped in nutlet. Ovary 2-3-carpellate, unilocular, with a single ovule. Nutlets usually a hard, a 2 or 3 -sided nutlet, rarely with a succulent or corky exocarp, surface smooth or variously minutely patterned, sometimes partially or completely enclosed by an enlarged basal prophyll (utricle), sometimes with a cup-like hypogynous disk at base.

Cyperaceae comprises ca. 104 genera and ca. 5000 species (Goetghebeur 1998). The family is nearly cosmopolitan but does not occur in Antarctica.

Cyperaceae can be recognised by the minute bisexual or unisexual flowers with the perianth reduced to small bristles or blades or absent, the flowers subtended by small bracts (glumes or floral bracts) these being aggregated into inflorescence units (spikelets and spicoids) which in turn are aggregated larger partial and full inflorescences. The fruit is a small, hard, 1-seeded nutlet.

The closest relatives to Cyperaceae are Juncaceae and Thurniaceae in the order Cyperales (Dahlgren et al. 1985, Simpson 1995). Gramineae, which shares some characteristics of Cyperaceae such as wind pollination and reduced floral structure, has often been placed near to Cyperaceae, but is now thought to be more distantly related (Linder \& Kellogg 1995, Simpson 1995).

${ }^{1}$ Royal Botanic Gardens, Kew, Richmond, Surrey, TW9 3AB, U.K. 
Inflorescence structure in Cyperaceae is difficult to interpret due to its highly reduced nature. Consequently, the terminology used in describing parts of the inflorescence is confusing with several terms often being applied to the same structure. In addition, several terms are also used in the Poaceae but they do not always relate to the same structure in both families. In this account an attempt has been to keep terminology as simple as possible. Definitions of the terms used are given in the Glossary below.
For accurate identification of Cyperaceae good fruiting material should be used wherever possible. Indeed this is essential in certain genera, such as Fimbristylis and Scleria. It is also important to have underground parts as these may be diagnostic for some species. Care is needed when counting the number of stigmas as these are easily broken off. Several should be observed from the same specimen. Care is also needed when counting the stamens. Anthers break off easily leaving the filaments partially hidden within the glumes. Always check that filaments are present.

\section{Glossary}

Terms which are italicised within each definition are themselves defined elsewhere in the Glossary.

Acuminate. Gradually narrowed to a long fine point.

Acute. Abruptly narrowed to a short point.

Biconvex. Two-sided, the sides convex.

Cancellate. Having the appearance of a lattice.

Capitate. Head-like inflorescence, without any apparent branching.

Compound. Applied to an inflorescence or partial inflorescence where there are two orders of branching, i.e. primary and secondary.

Compressed-trigonous. Three-sided, but distinctly flattened and thus appearing to be two-sided.

Conical. Cone-shaped, being wider at the base than the apex; here it is used as the 3-dimensional equivalent of lanceolate.

Connective. Tissue connecting the pollen sacs of an anther. Sometimes it extends beyond the apex of the pollen sacs to form a prominent tip to the anther.

Contraligule. Membranous, ligule-like structure at the apex of the leaf-sheath on the side of the culm facing away from the leaf-blade.

Coriaceous. Having a leathery texture.

Culm. Stem supporting the inflorescence.

Cylindric. Cylinder-shaped.

Decompound. Applied to an inflorescence or partial inflorescence where there are three or more orders of branching, i.e., primary, secondary and tertiary.

Deltoid. Triangular in outline.

Disk. Three-lobed structure occurring at the base of the nutlet in Scleria, Calyptrocarya and Becquerelia. In some species it may be indistinct.

Filiform. Thread-like.

Fimbriate. With a margin divided into a fringe.

Floral bract. Membranous scale-like structure in the spicoid-type inflorescence unit each of which subtends a male flower comprising a single stamen only. The lowest two floral bracts are usually have a keel and are opposite.

Globose. Rounded, resembling a ball. 
Glume. Membranous to coriaceous scale-like structure subtending individual flowers.

Hyaline. Transparent and usually colourless.

Imbricate. Tightly overlapping.

Involucral bract. Bract or bracts occurring at the point where the inflorescence arises from the culm. Vary from being leaf-like to glume-like or setaceous.

Keel. Used here for the midrib of a glume or floral bract.

Lanceolate. Lance-shaped, i.e. broadest below the middle and gradually tapering above.

Ligule. Membranous tissue or fringe of hairs occurring at the apex of the leaf sheath on the inner side at the point where it joins the leaf-blade.

Mucronate. Terminating in a short stiff point.

Nutlet. Hardened, usually minute, one-seeded fruit, the surface of which may be smooth to variously patterned and a diagnostic character for many species. Often called an achene in literature on Cyperaceae.

Ob. (prefix) Used to indicate inversion of a shape, e.g. obdeltoid, obovoid, oblanceolate.

Obtuse. Blunt.

Orbicular. Circular.

Ovate. Egg-shaped in two-dimensional outline.

Ovoid. Egg-shaped in three dimensions.

Paniculate. Inflorescence partial inflorescences arising at intervals along the main inflorescence axis.

Partial inflorescence. Primary branches of an inflorescence.

Perianth segments. Small bristle-like or scale-like structures at the base of the nutlet. Presumed to be the remnants of a fully developed perianth.

Plicate. Folded longitudinally.

Prophyll. Two-keeled structure at the base of a branch within an inflorescence. It may be glumelike or tubular.

Puncticulate. Dotted.

Reticulate. Forming a network.

Retrorse. Turned backwards.

Rhizome. Underground stem which may be short, often giving the plant a tufted habit, or long-creeping.

Rugose. Wrinkled.

Setaceous. Bristle-like.

Simple. Applied to an inflorescence or partial inflorescence where there is only order of branching, i.e. primary branching.

Spicoid. The ultimate inflorescence unit in Cyperaceae Tribe Hypolytreae. Has a much reduced axis and appears flower-like. It comprises 2-6 floral bracts each subtending a male flower. The whole structure is terminated by a female flower.

Spicoid bract. A glume-like bract which subtends the spicoid.

Spike. An aggregation of spikelets or spicoids; sometimes the whole structure is similar in appearance to a spikelet (in Mapania and Hypolytrum).

Spikelet. The ultimate inflorescence unit in most genera of Cyperaceae. Has an elongated or reduced axis with 1-many glumes, each glume subtending a bisexual or unisexual flower.

Stolon. In Cyperaceae this term is applied to a thin underground branch arising from the rhizome or base of the culm. Each stolon terminates in an aerial shoot. 
Style-base. A variously-shaped portion at the base of the style which is persistent on the mature nutlet in some genera.

Terete. Circular in cross-section.

Tomentose. Thickly covered with short hairs.

Trigonous. Three-sided, with the margins blunt and rounded. Applied here to the culm and nutlet.

Triquetrous. Three-sided with the margins acute. Applied here to the culm and nutlet.

Umbel-like. Inflorescence in which the primary branches more-or-less arise from the same point, the inflorescence being subtended by 1 -several involucral bracts.

Verruculose. Covered with small wart-like outgrowths.

\section{Key to the genera of Cyperaceae in Reserva Ducke}

1. Inflorescence comprising small units (spicoids) with 2 opposite, keeled, often ciliate scales (floral bracts) at the base often enclosing a further 2-6 scales, each unit subtended and usually hidden or partially hidden by a glume-like bract.

2. Inflorescence umbel-like; stamens 7-8 per spicoid.

4. Diplasia

2. Inflorescence paniculate or capitate; stamens 1-3 per spicoid

3. Inflorescence capitate; spicoids with 4-6 floral bracts 10. Mapania

3. Inflorescence paniculate; spicoids with 2(-3) floral bracts 8. Hypolytrum

1. Inflorescence various but not as above.

4. All flowers unisexual.

5. Female spikelets subtended at base by 3 sterile spikelets; nutlet very tightly enclosed by a delicate, membranous sac

2. Calyptrocarya

5. Female spikelets without sterile spikelets at base; nutlet not enclosed by a membranous sac.

6. Contraligule present in leaf sheath apex; disk at base of nutlet not spongy

6. Contraligule absent; disk at base of nutlet spongy

1. Becquerelia

4. At least some flowers bisexual.

7. Glumes increasing in length towards the apex of the spikelet.

8. Inflorescence paniculate (in Ducke species); perianth segments scabrid below, ciliate or fimbriate above 11. Pleurostachys

8. Inflorescence capitate (in Ducke species); perianth segments scabrid or rarely smooth 12. Rhynchospora

7. Glumes \pm equal in length (but often with 1-3 smaller glumes at base of spikelet).

9. Perianth segments present.

10. Leaves reduced to bladeless sheaths; inflorescence a single spikelet

5. Eleocharis

10. Leaf blades present; inflorescence with more than one spikelet

7. Fuirena

9. Perianth segments absent.

11. Glumes spirally arranged; style jointed with ovary and clearly demarcated from it 6. Fimbristylis

11. Glumes 2-ranked; style continuous with ovary and not demarcated from it.

12. Stigmas 2; nutlet 2-sided

9. Kyllinga

12. Stigmas 3; nutlet 3-sided

3. Cyperus 


\section{Becquerelia}

Perennial herbs; rhizomatous or rarely stoloniferous. Culms triquetrous. Leaves basal and cauline, 3-ranked; ligule 0; contraligule absent. Involucral bracts leaf-like. Inflorescence paniculate; partial inflorescences corymbose or rarely capitate. Spikelets unisexual; male spikelets comprising $c a$. 5 glumes, the lower subtending a flower; female spikelets comprising $c a .10$ sterile glumes and a single terminal flower. Perianth segments 0. Stamens 1 per flower. Stigmas 3. Nutlets depressed-globose, smooth, rugulose or tuberculate with a spongy cup-like disk at base.

Genus of five species, central and South America.

1.1 Becquerelia cymosa subsp. merkeliana (Nees) T. Koyama, Mem. N.Y. Bot. Gard. 17(1): 29. 1967.

Becquerelia merkeliana Nees, in Mart., Fl. bras. 2(1): 191. 1842.

Perennial. Culm $39 \mathrm{~cm}$ long, $6.5 \mathrm{~mm}$ wide, smooth. Leaves: blade linear, $68 \mathrm{~cm}$ long, 1.1-1.4 cm wide, gradually narrowed, acuminate, flat to v-shaped in cross-section; sheath $13-16 \mathrm{~cm}$ long, green to pale brown. Involucral bracts $7-43 \mathrm{~cm}$ long, the lowest bract longest. Inflorescence $40 \times 5.5 \mathrm{~cm}$; nodes 6 , each subtending 1-2 corymbose partial inflorescences; partial inflorescences 3-4 $\times 4 \mathrm{~cm}$, compound; primary branches $0.5-2 \mathrm{~cm}$ long; secondary branches $0.2-1 \mathrm{~cm}$ long, terminating in clusters of several spikelets. Male spikelets 1-2 below female spikelets, narrowly lanceolate, 3-3.5 $\times$ $0.2 \mathrm{~mm}$. Female spikelets ovate, 3-5-4.1 $\times$ $1.3-1.5 \mathrm{~mm}$. Anthers $1 \mathrm{~mm}$ long. Nutlets $2 \times$ $1.8-2 \mathrm{~mm}$, whitish, rugulose-reticulate.

Tropical South America.

17.IX.1958 (fl) Coêlho, D. 7 (INPA).

Additional specimen examined: BRAZIL. AMAZONAS: Igarapé de Tarumã Assunção, $P$. A. C. L. 383 (INPA); Boa Vista road, $48 \mathrm{~km} \mathrm{~N}$ of Manaus, 21.IX.1980 Lowe 3996 (INPA K).

\section{Calyptrocarya}

Perennial herbs; rhizomatous or stoloniferous. Culms trigonous to triquetrous. Leaves basal and cauline, 3-ranked; ligule 0, contraligule sometimes present. Involucral bracts leaf-like. Inflorescence capitate or cymose-paniculate with distant nodes each subtending a single partial inflorescence; partial inflorescence umbel-like, each with several rayed globose spikelet clusters. Spikelets unisexual or sterile. Male spikelets comprising several glumes. Female spikelets comprising a single, apparently terminal female flower, very tightly enclosed by a delicate, membranous sac. Sterile spikelets lateral, in 3 at the base of the female spikelet, each comprising a few empty glumes, the spikelets subtended by 3 glume-like bracteoles. Perianth segments 0. Stamen 1 per male flower. Stigmas $2-3$. Nutlets 2 -sided to terete-trigonous, surface bony, white.

Genus of eight species, central and South America.

\section{Key to the species of Calyptrocarya in Reserva Ducke}

1. Spikelet clusters up to $3 \mathrm{~mm}$ wide; nutlets up to $1.5 \mathrm{~mm}$ wide 2. C. glomerulata

1. Spikelet clusters $5 \mathrm{~mm}$ or more wide; nutlets $1.8 \mathrm{~mm}$ or more wide.

2. Leaf blade abruptly narrowed at apex, narrowed into a pseudopetiole towards base 1. C. bicolor

2. Leaf blade gradually narrowed at apex, not narrowed into a pseudopetiole towards base 2 . 3. C. poepiggiana 
2.1 Calyptrocarya bicolor (H. Pfeiff.) T. Koyama, Mem. N.Y. Bot. Gard. 17(1): 43. 1967.

Becquerelia bicolor H. Pfeiff., Fedde, Repert. 18: 381. 1922.

Perennial. Rhizome short-creeping. Culms 3-12 cm long, 1-1.4 mm wide, triquetrous, scabrid towards apex. Leaves: blade linear, linear-elliptic, 7-20 cm long, 5$12 \mathrm{~mm}$ wide, abruptly narrowed at apex, acute, flat, green above, usually mid- to dark reddish below, narrowed below into a pseudopetiole towards base; sheath $1-4 \mathrm{~cm}$ long, dark reddish. Lowest involucral bract up to $17 \mathrm{~cm}$ long, upper bracts shorter. Inflorescence cymose-paniculate, $4-14 \mathrm{~cm}$ long, nodes 3-4; partial inflorescence with rays $0.5-2 \mathrm{~cm}$ long. Spikelet clusters $4-6 \times$ 5-7 mm. Nutlets 2 -sided, broadly obovate, $1.8-2 \times 1.8-2 \mathrm{~mm}$.

Tropical South America.

Forest.

11.II.1995 (fr) Costa, M. A. S. et al. 149 (INPA); 13.V.1996 (fl) Costa, M. A. S. et al. 512 (INPA); 6.VI.1988 (fl) Santos, J. L. 952 (INPA K MG MO NY RB SP); 4.VI.1995 (fl) Sothers, C. A. 495 (INPA); 28.IV.1994 (fl) Vicentini, A. et al. 520 (INPA); 13.V.1996 (fl) Costa, M. A. S. et al. 512 (INPA); 6.VI.1988 (fl) Santos, J. L. 952 (INPA K MG MO NY RB SP); 4.VI.1995 (fl) Sothers, C. A. 495 (INPA); 28.IV.1994 (fl) Vicentini, A. et al. 520 (INPA).

Calyptrocarya bicolor is distinguished by having the leaf blade narrowed into a pseudopetiole towards its base and a distinct reddish coloration to the undersides of the leaves.

2.2 Calyptrocarya glomerulata (Brongn.) Urb., Symb. Ant. 2(1): 169. 1900.

Becquerelia glomerulata Brongn., in Duperry, Voy. Coq. 2: 163. 1829.

Calyptrocarya angustifolia Lindl. \& Nees ex Kunth, Enum. Pl. 2: 364. 1837.

Calyptrocarya fragifera sensu Kunth, Enum. Pl. 2: 364.1837 non (Rudge) Nees.

Calyptrocarya intermedia C.B. Clarke, Kew Bull. Add. Ser. 8: 66, 135. 1908.

Perennial. Rhizome short. Culms tufted, $7.5-9 \mathrm{~cm}$ long, $0.8-1 \mathrm{~mm}$ wide, triquetrous. Leaves: blade narrowly linear, up to $21 \mathrm{~cm}$ long, 2-3 mm wide, gradually acuminate at apex, flat, green above, green or brownish below; sheath 4-6.5 cm long, reddish-brown. Lowest involucral bract $20 \mathrm{~cm}$ long, upper bracts shorter. Inflorescence cymosepaniculate, 7-8 cm long, nodes 3-4; partial inflorescence with rays $0.5-0.7 \mathrm{~cm}$ long. Spikelet clusters $2 \times 2-3 \mathrm{~mm}$. Nutlets 2 -sided, broadly obovate, $1-1.5 \times 1.5 \mathrm{~mm}$.

Southern Mexico to Brazil.

Forest.

6.VII.1993 (fl) Ribeiro, J. E. L. S. et al. 899 (INPA $\mathrm{KNY}$ ).

Calyptrocarya glomerulata can be recognised by its linear, gradually narrowed leaves, small spikelet clusters and small nutlets.

2.3 Calyptrocarya poepiggiana Kunth, Enum. Pl. 2: 364. 1837.

Calyptrocarya martii Nees, in Mart., Fl. bras. 2(1): 195. 1842.

Perennial. Rhizome short-creeping. Culms 3-12 cm long, 0.8-0.9 mm wide, triquetrous, scabrid towards apex. Leaves: blade narrowly linear, 11-40 cm long, 5-7 mm wide, gradually narrowed at apex, acute, flat, green above, usually mid- to dark reddish below, not narrowed into a pseudopetiole towards base; sheath 1-4 cm long, dark reddish. Lowest involucral bract up to $43 \mathrm{~cm}$ long, upper bracts shorter. Inflorescence cymose-paniculate, 7-15 cm long; nodes 34 ; partial inflorescence with rays $0.8-3.3 \mathrm{~cm}$ long. Spikelet clusters $4-6 \times 5-6 \mathrm{~mm}$. Nutlets 2 -sided, broadly obovate, $2 \mathrm{~mm} \times$ $1.8 \mathrm{~mm}$.

Tropical South America, particularly the tropical Andean region.

Forest.

14.II.1996 (fr) Campos, M. T. V. A. et al. 489 (INPA KMGNY SP).

Calyptrocarya poepiggiana also has linear, gradually narrowed leaves, but the spikelet clusters and nutlets are similar to $C$. bicolor.

\section{Cyperus}

Annual or perennial herbs; rhizomatous or stoloniferous. Culms terete to trigonous. Leaves basal, 3-ranked, rarely without blade; 
ligule 0 . Involucral bracts leaf-like. Inflorescence terminal, umbel-like and 1-3-times branched, with the ultimate branches terminating in 1 or more spikes or finger-like cluster of spikelets, more rarely spikes or spikelet clusters sessile or inflorescence capitate. Spikelets linear to oblong or elliptic, laterally flattened to subterete; axis straight or zigzag, deciduous or persistent. Glumes \pm equal in length, 2-ranked, deciduous or persistent, sides membranous to chartaceous or coriaceous, nerves 0 -several, keel acute to rounded. Flowers bisexual. Perianth segments 0 . Stamens 1-3. Stigmas (1-)3; style continuous with ovary. Nutlets usually 3 -sided, trigonous, sometimes triquetrous or dorsiventrally compressed.

About 500 species, \pm cosmopolitan, but particularly abundant in the tropics.

\section{Key to the species of Cyperus in Reserva Ducke}

1. Spikelets in finger-like clusters; glumes ovate-orbicular 1. C. laxus

1. Spikelets aggregated into spikes; glumes ovate to lanceolate.

2. Culms papillose; spikelets dark coppery brown; nutlets $1.5 \mathrm{~mm}$ long 2. C. ligularis

2. Culms smooth or scabrid; spikelets greenish, yellowish or whitish; nutlets up to $1.1 \mathrm{~mm}$ long

3. Culms smooth; spikelets whitish, in dense clusters within the spike..... 3. C. luzulae

3. Culms smooth to retrorsely scabrid; spikelets greenish or yellowish in loose clusters within the spike 4. C. surinamensis

3.1 Cyperus laxus Lam., Ill. Gen. 1: 146. 1791; J. Raynal, Adansonia 2, 17(3): 277. 1978.

C. diffusus Vahl, Enum. Pl. 2: 321. 1805.

Perennial. Rhizome short. Culms \pm tufted, $36 \mathrm{~cm}$ long, 1.7-1.9 mm wide, trigonous to subtriquetrous, smooth. Leaves: blade linear, 15-30 cm long, 5-5.4 mm wide, abruptly acute, flattish to plicate; sheath $4 \mathrm{~cm}$ long, pale green to rusty or purplish brown. Involucral bracts 4-12, unequal, the longest up to $50 \mathrm{~cm}$. Inflorescence umbel-like, 1-2 times branched; primary branches $10-12,2-12 \mathrm{~cm}$ long; secondary branches $0.5-1.5 \mathrm{~cm}$ long. Spikelets in open finger-like clusters of (1-)29, oblong, 3-11 × 1.5-2.5 mm. Glumes 6-20 per spikelet, ovate-orbicular, 1.5-2 $\times 1.5-2 \mathrm{~mm}$, obtuse, mucronate, awn $0.2-0.5 \mathrm{~mm}$ long, sides membranous, indistinctly nerved, greenish tinged with pale or reddish-brown, keel greenish. Stamens 3; anthers $0.8-1 \mathrm{~mm}$ long. Stigmas 3. Nutlets ellipsoid, trigonous, 1.2$1.5 \times 0.7-0.8 \mathrm{~mm}$, pale brown becoming blackish brown, indistinctly puncticulate.

Pantropical.

Forest or forest margins. 26.IV.1981 (fl) Lowe, J. 4105 (INPA). Additional specimens examined: BRAZIL. RORAIMA: Boa Vista, Reserva Ecologica de Maracá
7.III.1987 Harley 24735(K); AMAZONAS: Manaus, near Praia Dourada 23.VII.1980 Lowe 3949 (INPA, K).

Cyperus laxus is distinguished by spikelets in finger-like clusters and ovateorbicular glumes.

3.2 Cyperus ligularis L., Syst. Nat 10, 2 : 867. 1759; Nees in Mart., Fl. bras. 2(1): 42. 1842; Kük. in Engl., Pflanzenr. 4(20), 101 Heft: 474. 1936.

Mariscus ligularis (L.) Urb., Symb. Antill. 2(1): 165. 1900.

Perennial. Rhizome short. Culms \pm tufted 50-100 cm long, 4-5 mm wide, trigonous, papillose. Leaves: blade linear, up to $80 \mathrm{~cm}$ long, 5-10 $\mathrm{mm}$ wide, longacuminate, flattish to folded; sheath $12-19 \mathrm{~cm}$ long, mid-brown to dark reddish-brown. Involucral bracts 8 , unequal, the longest up to $65 \mathrm{~cm}$ long. Inflorescence umbel-like, 1(2)-times branched; primary branches up to 10, 1-6 cm long; secondary branches (when developed) up to $3 \mathrm{~cm}$ long. Spikes 4-7 per inflorescence branch, cylindric to subglobose, $1.1-3.5 \mathrm{~cm}$ long, the uppermost spike longest. Spikelets numerous, densely crowded, oblongelliptic, 3-7 × 1-2.5 mm, dark coppery brown. Glumes 4-7 per spikelet, ovate, 2- 
$2.5 \mathrm{~mm}$ long, 1-2 mm wide, acute, very shortly mucronate, sides membranous, 4-5nerved, dark brown with reddish tinge, keel greenish or brown. Stamens 3; anthers $0.5 \mathrm{~mm}$ long. Stigmas 3. Nutlets ellipsoid, trigonous, $1.5 \times 0.6 \mathrm{~mm}$, dark purplish brown, puncticulate.

Florida, W. Indies, tropical America, Africa, Indian Ocean islands.

Disturbed areas in forest.

26.X.1977 Keel \& Balick 203 (K NY); 14.XII.1966 (fl) Prance, G. T. et al. 3637 (INPA KNY).

Additional specimens examined: BRAZIL. AMAZONAS: Manaus, Praia Dourada 23.V.1981 Lowe 4193 (K); Manaus-Itacoatiara road, km 63 17.XII.1968 Prance et al. 9058 (K).

Cyperus ligularis has papillose culms and spikes comprising numerous dark coppery brown spikelets.

3.3 Cyperus luzulae (L.) Retz., Obs. Bot. 4: 11. 1786; Kük. in Engl., Pflanzenr. 4(20), 101 Heft: 170. 1936.

Scirpus luzulae L., Syst. Nat. 10, 2: 868. 1759.

Perennial. Rhizome short. Culms tufted, 28-51 cm long, 1.6-2.8 mm wide, trigonous, smooth. Leaves: blade linear, 20$30 \mathrm{~cm}$ long, 3.5-4.5 mm wide, gradually acuminate, flattish to folded; sheath 3$7.5 \mathrm{~cm}$ long, pale to mid-brown. Involucral bracts 5-10, unequal, the longest up to $36 \mathrm{~cm}$. Inflorescence umbel-like, oncebranched; primary branches $6-11,1.1-5 \mathrm{~cm}$ long. Spike 1 per inflorescence branch, ovoid to ovoid-cylindric, $0.8-1.5 \mathrm{~cm}$ long. Spikelets in numerous, densely crowded clusters within spike, ovate, $2-4.5 \times 1-2 \mathrm{~mm}$, whitish. Glumes 6-20 per spikelet, deciduous, lanceolate to ovate-lanceolate, 1.5-2 × 0.4-0.8 mm, obtuse, mucronulate, sides membranous, 1-2-nerved, whitish, keel similar. Stamen 1; anther $0.7 \mathrm{~mm}$ long. Stigmas 3. Nutlets narrowly oblongellipsoid, trigonous to subterete, $1-1.1 \times$ $0.3 \mathrm{~mm}$, brown to blackish, \pm smooth.

Subtropical and tropical America.

Open, damp places.
17.I.1995 (fl) Costa, M. A. S. et al. 99 (INPA K MG MO NY R RB SP U); 8.VI.1995 (fl) Costa, M. A. S. \& Silva, C. F. 302 (BM G INPA K MBM MG UB UEC US); 29.XI.1976 (fl) Mendonça, S. \& Shima, D. 24 (INPA).

Additional specimens examined: BRAZIL. AMAZONAS: Boa Vista road, $48 \mathrm{~km}$ N of Manaus 21.IX.1980 Lowe 3995 (K); road ZF2, 14 km off Boa Vista road, $62 \mathrm{~km}$ NNW of Manaus 21.VI.1981 Lowe 4316 (K); Itacoatiara, $275 \mathrm{~km}$ E of Manaus 9.VI.1981 Lowe 4257 (K).

Cyperus luzulae is recognisable by its numerous whitish spikelets occurring in densely crowded clusters in each spike.

3.4 Cyperus surinamensis Rottb., Descr. Pl. Rar.: 20. 1772; Nees in Mart., Fl. bras. 2(1): 21. 1842; Kük. in Engl., Pflanzenr. 4(20), 101 Heft: 174. 1936.

Annual or perennial. Rhizome short. Culms tufted, 26-43 cm long, 1.2-1.5 mm wide, trigonous to subterete, smooth to retrorsely scabrid. Leaves: blade linear, 13$30 \mathrm{~cm}$ long, 1.3-2.5 mm wide, gradually acuminate, folded; sheath 3-7 cm long, greenish to pale brown. Involucral bracts 5, unequal, the longest $14-18 \mathrm{~cm}$ long. Inflorescence umbel-like, 1-2-times branched; primary branches 6-12, 0.7-4 cm long; secondary branches when present $0.3-$ $0.6 \mathrm{~cm}$ long. Spikes half-globose to \pm globose, $0.6-0.8 \times 0.8-1 \mathrm{~cm}$. Spikelets $8-40$ per spike, in loose clusters, lanceolate to linear-oblong, $5-7 \times 1.7-1.8 \mathrm{~mm}$, greenish to yellowish. Glumes 15-60 per spikelet, deciduous, lanceolate to ovate-lanceolate, $1.3-1.5 \times 0.2-$ $0.3 \mathrm{~mm}$, obtuse to acute, mucronulate, sides membranous, 1-2-nerved, greenish to yellowish, keel similar. Stamen 1; anther $0.5 \mathrm{~mm}$ long. Stigmas 3. Nutlets narrowly ellipsoid, $0.8 \times 0.2-0.3 \mathrm{~mm}$, mid-brown, minutely papillose.

Subtropical and tropical America.

Moist, open areas.

8.VI.1995 (fr) Costa, M. A. S. \& Silva, C. F. 301 (INPA K MG MONY R RB SP U).

Additional specimen examined: BRAZIL. AMAZONAS: Manaus, 8 Tr. A, Jardim Haydea 3.IV.1981 Lowe 4064 (K). 
Cyperus surinamensis is closely related to $C$. luzulae but differs in often having scabrid culms and greenish to yellowish spikelets that are in looser clusters within the spikes.

\section{Diplasia}

Robust, rhizomatous perennial herbs. Rhizome thick, woody. Culms loosely tufted, central, erect. Leaves basal and cauline, leathery; ligule 0 . Involucral bracts leaf-like, unequal. Inflorescence umbel-like, 2-3-times branched. Spikes in clusters of 2-7 at tips of branches, sessile or shortly pedunculate, narrowly cylindric comprising many spirally imbricate, leathery, glume-like bracts (spicoid bracts) each subtending a partial inflorescence (spicoid) with a much reduced axis. Spicoids comprising a naked, terminal female flower and 5-6 scale-like floral bracts, the lowest 2 floral bracts opposite, keeled, ciliate on keel, the upper bracts \pm connate, each subtending a male flower. Perianth segments 0. Stamens 1-3 per male flower. Stigmas 2. Nutlets ellipsoid, obtuse, slightly compressed, smooth.

Monotypic genus.

4.1 Diplasia karataefolia Rich. in Pers., Syn. Pl. 1: 70. 1805: Nees in Mart., Fl. bras. 2(1): 70. 1842 .

Perennial. Culms to $3 \mathrm{~m}$ long, $6 \mathrm{~mm}$ wide, trigonous, smooth. Leaves: blade linear, up to $3 \mathrm{~m}$ long, $4 \mathrm{~cm}$ wide, gradually narrowed, acuminate leathery; sheath 10$12 \mathrm{~cm}$ long, mid-brown. Involucral bracts 5 , the longest up to $50 \mathrm{~cm}$ long. Inflorescence: primary branches 7 or more, up to $16 \mathrm{~cm}$ long; secondary branches $1.5-5 \mathrm{~cm}$ long. Spikes $1.5-3.5 \mathrm{~cm} \times 2-4.5 \mathrm{~mm}$, pale to mid-brown. Spicoid bracts ovate, $5.4-5.5 \times 3.2 \mathrm{~mm}$, obtuse, pale to mid-brown, margins paler, keel greenish to brown. Spicoids \pm equalling the spicoid bracts. Floral bracts 3.5-4 mm long, $1 \mathrm{~mm}$ wide. Stamens $7-8$ per spicoid. Nutlets 6-6.2 $\times 4.2-5 \mathrm{~mm}$, mid-brown.

Tropical South America. Forest.
24.V.1967 (st) Albuquerque, B. W. P. \& Elias, J. 63 (INPA); 9.XII.1994 (fr) Costa, M. A. S. \& Nascimento, J. R. 42 (INPA); $12 . X .1966$ (fl) Prance, G. T. et al. 2632 (INPA); 1.VII.1994 (fl) Ribeiro, J. E. L. S. 1335 (K); 28.IV.1964 (fl) Rodrigues, W. \& Loureiro, A. 5801 (INPA); 3.II.1965 (fl) Rodrigues, W. \& Monteiro, O. P. 6853 (INPA); 29.I.1998 (fl) Souza, M. A. D. et al. 541 (INPA).

Additional specimen examined: BRAZIL. AMAZONAS: $c a .20 \mathrm{~km}$ from Manaus 27.X.1989 Bogner 2019 (K).

Diplasia karataefolia is unmistakable, being a very robust plant with large leathery leaves and an umbel-like inflorescence.

\section{Eleocharis}

Annual or perennial herbs. Rhizome short or creeping. Culms terete or angular, sometimes transversely septate. Leaves reduced to bladeless sheaths; ligule 0 . Involucral bracts $1 * 2$, glume-like. Inflorescence a single, terminal spikelet. Spikelet ovoid, ellipsoid or cylindric. Glumes several to many per spikelet, \pm equal in length, usually spirally imbricate, rarely 2-ranked. Flowers bisexual. Perianth segments up to 8 , bristle-like, sometimes 0 . Stamens 1-3. Stigmas 2-3. Nutlets trigonous or biconvex, mostly obovate; surface smooth, reticulate (cancellate), pitted, longitudinally grooved or transversely ridged; style-base persistent on nutlets.

About 180 species occurring in tropical and temperate regions worldwide.

5.1 Eleocharis filiculmis Kunth, Enum. Pl. 2: 144. 1837; Svenson, Rhodora 39: 266. 1937.

Annual or perhaps short-lived perennial. Culms tufted, 20-26 cm long, 0.6-0.8 mm wide, 4-angled with 1-2 \pm central channels down each side of culm. Sheaths $1.3-3 \mathrm{~cm}$ long, apex acute to subobtuse, dark reddish. Spikelets ellipsoid, to ellipsoid-cylindric, terete, 5-7 × 2-3 mm. Glumes many per spikelet, oblong, 1.8-1.9 mm long, $1 \mathrm{~mm}$ wide, obtuse to rounded, sides membranous with broad membranous margin, pale brown minutely reddish-striate, midrib mid-brown. Perianth segments 6-7, shorter than nutlet. Stamens 2; 
anthers $0.9 \mathrm{~mm}$ long. Stigmas 3. Nutlets obovoid, trigonous, $0.9 \times 0.5 \mathrm{~mm}$, apex rounded, light brown; surface smooth, somewhat shiny; style-base pyramidal.

Tropical America.

Wet places.

17.I.1995 (fl) Costa, M. A. S. \& Nascimento, J. R. 101 (INPA K MG MO NY R RB SP U); 26.IV.1981 (fr) Lowe, J. 4107 (INPA K UIH).

Eleocharis filiculmis can be distinguished by leaves that are reduced to bladeless sheaths, a single spikelet on each culm and nutlets with a prominent pyramidal style-base.

\section{Fimbristylis}

Annual or perennial herbs. Rhizome short creeping. Culms usually tufted, angled, trigonous or flattened. Leaves basal, bladed or reduced to bladeless sheaths; blades linear to filiform, often canaliculate, often cellularreticulate on upper surface; ligule sometimes present, pubescent or membranous. Involucral bracts leaf-like, setaceous or glume-like. Inflorescence umbel-like and 1-3 times branched or capitate or a single spikelet. Spikelets mostly ovoid or ellipsoid, terete, angular or \pm laterally flattened. Glumes few to many per spikelet, spirally arranged. Flowers bisexual. Perianth segments 0 . Stamens 1-3. Stigmas 2-3; style jointed with ovary and clearly demarcated from it; style base not persistent on nutlets. Nutlets trigonous or biconvex, surface variously patterned.

Genus of 200 species mostly in the tropics and subtropics, with the highest number in S. Asia, Indo-China and Malesia.

6.1 Fimbristylis dichotoma (L.) Vahl, En. Pl. 2: 287.1806.

Scirpus dichotomus L., Sp. Pl. 1: 50.1753.

Annual or short-lived perennial. Rhizome short. Culms tufted, $18-50 \mathrm{~cm}$ long, 0.5-0.8 mm wide, trigonous, glabrous, smooth. Leaves basal; blade narrowly linear, 4-16 cm long, $1.3 \mathrm{~mm}$ wide, obtuse, flattish; sheath 2$12 \mathrm{~cm}$ long, sparsely pubescent; ligule a fringe of dense hairs. Involucral bracts 2-7, the longest 1-2 leaf-like, $2-8 \mathrm{~cm}$ long. Inflorescence umbel-like, 1-2 times branched, open, 2-8 $\times$ $1.5-5 \mathrm{~cm}$; primary branches $2-5,0.7-6 \mathrm{~cm}$ long. Spikelets $2-14$ per inflorescence, solitary, ovoid to ovoid-ellipsoid, 3-7 mm long, 1.5$2.5 \mathrm{~mm}$. Glumes many per spikelet, \pm equal in length, spirally arranged, broadly ovate to suborbicular, $1.5-3 \times 1.5-2.2 \mathrm{~mm}$, subacute, mucronate, sides thinly chartaceous, nerves 0 , mid-brown to dark reddish-brown, margins palehyaline, keel obtuse, greenish to pale brown. Stamen 1. Stigmas 2. Nutlets obovate to broadly obovate, biconvex, $0.7 \times 0.6 \mathrm{~mm}$, maturing cream or pale brown, deeply cancellate with 5-6 rows of transversely oblong epidermal cells on each side.

Tropical (India, type), subtropical and warm-temperate regions worldwide.

Open, damp places.

17.I.1995 (fl) Costa, M. A. S. \& Nascimento, J. R. 102 (INPA K MG MO NY R RB SP U); 26.IV.1981

(fl) Lowe, J. 4104 (INPA).

Fimbristylis dichotoma is a common weedy species throughout the tropics. The best character for distinguishing it is the nutlet which has 5-6 rows of transversely oblong epidermal cells on each side. It also has an umbel-like inflorescence with spirally arranged glumes in each spikelet.

\section{Fuirena}

Annual or perennial herbs. Rhizome short or creeping. Culms (3-)4-5-angular, nodose. Leaves mostly cauline; blade pubescent or glabrous, 3-5-nerved; sheaths closed; ligule 0 . Involucral bracts leaf-like, sheathing at base, equalling or longer than inflorescence. Inflorescence paniculate, with few to many clusters of sessile spikelets at few to several nodes. Spikelets with many glumes. Glumes \pm equal in length, spirally imbricate, pubescent outside, usually shortly awned, 13-nerved, the lowest 1-3 empty. Flowers bisexual. Perianth segments 3-6 in 1-2 whorls each of 3 segments, outer whorl of simple bristles, sometimes absent, inner whorl of 
bristles, blades or claws. Stamens 2-3. Stigmas 3 ; style continuous with ovary. Nutlets trigonous to triquetrous, apex beaked, base cuneate to stipitate, smooth to trabeculate.

Genus of $c a .30$ species in the tropics, the largest number being in Africa.

7.1 Fuirena umbellata Rottb., Descr. \& Ic. Rar.: 70. t.19 fig. 3. 1773; Nees in Mart., Fl. bras. 2(1): 107. 1842.

Perennial. Culms up to $60 \mathrm{~cm}$ long, 6$9 \mathrm{~mm}$ wide, pubescent below inflorescence. Leaves 5-7, cauline; blade lanceolate to linear-lanceolate, $8-12 \mathrm{~cm}$ long, 5-15 mm wide, acute, ciliate at base; sheath $2-5 \mathrm{~cm}$ long, usually glabrous. Inflorescence with 3-12 clusters of spikelets, peduncles whitishpubescent. Spikelets ovoid or ovoid-ellipsoid, \pm squarrose, 4-8 $\times 2 \mathrm{~mm}$, acute, brownishgreen or dark brownish-green. Glumes obovate to ovate-elliptic, $2-2.5 \times 1.2-1.5 \mathrm{~mm}$, rounded, shortly pubescent, awn $0.8-1.2 \mathrm{~mm}$ long, often pilose. Perianth segments 3, in 1 whorl only, obovate or oblong, membranous, truncate, subsessile with a very short claw at base. Anthers $0.5-0.7 \mathrm{~mm}$ long. Nutlets obovoid to ellipsoid, $0.8-1.2 \times 0.6-0.7 \mathrm{~mm}$, shiny, smooth to obscurely wrinkled.

Pantropical.

Open, damp or wet places.

7.IX.1996 Assunção 384 (INPA K); 14.VI.1988 Santos 926 (INPA K).

Fuirena umbellata is easily distinguished by its cauline leaves. In addition, the perianth segments are obovate or oblong, a characteristic seen in any other Cyperaceae described here.

\section{Hypolytrum}

Stoloniferous or rhizomatous perennial herbs. Rhizome usually woody. Culms central or lateral, the latter with cataphylls at base. Leaves 3-ranked, basal or cauline; blade coriaceous, glabrous; pseudopetiole present or 0 ; ligule 0 . Involucral bracts leaflike, basal bract usually longest. Inflorescence usually paniculate, 1-2-times branched, more rarely capitate (not in Ducke taxa) with 1-many spikes. Spikes comprising many spirally imbricate glume-like bracts (spicoid bracts) each subtending a partial inflorescence (spicoid) with a much reduced axis. Spicoids comprising a naked, terminal female flower and 2(-3) scale-like floral bracts, all subtending a male flower, the lowest 2 floral bracts opposite, keeled. Perianth segments 0. Stamens 2 per spicoid, 1 per male flower. Stigmas 2-3. Nutlets sculptured, often with spongy conical apex.

About 50 species, pantropical.

8.1 Hypolytrum schraderianum Nees in Mart., Fl. bras. 2(1): 65. 1842; T. Koyama, Darwiniana 16(1-2): 56. 1970.

Perennial. Rhizome short-creeping. Culms central, 70-96 cm long, 2.2-3 mm wide, trigonous, smooth. Leaves basal and 2 cauline; blade linear, $90 \mathrm{~cm}$ long, 19-28 mm wide, gradually narrowed, 3-nerved; pseudopetiole absent; cauline sheaths $5-6 \mathrm{~cm}$ long, mid-brown to reddish-brown. Involucral bracts $2-3$, leaf-like, the longest $25-50 \mathrm{~cm}$ long. Inflorescence an open panicle, compound, broadly ovoid, $12-16 \times 14-16 \mathrm{~cm}$, comprising up to 12 primary branches each subtending up to 6 secondary branches terminating in tertiary branches subtending 13 sessile or shortly talked spikes. Spikes ovoid, ellipsoid to narrowly cylindric, 4-9 $\times 1-3 \mathrm{~mm}$, mid-brown. Spicoid bracts obovate, ellipticobovate $1.5-1.7 \times 0.9-1 \mathrm{~mm}$, rounded, midbrown. Spicoids \pm equalling or slightly exceeding the spicoid bracts. Floral bracts 2, \pm free, $1.4-1.8 \mathrm{~mm}$ long, keel ciliate. Stamens 2 per spicoid. Stigmas 3. Nutlets broadly ovoid to suborbicular, $1.5-2.1$ by $1-1 \mathrm{~mm}$, apex conical, irregularly longitudinally ridged.

Brazil, Colombia, Venezuela.

Forest.

16.III.1995 (fl) Costa, M. A. S. et al. 163 (INPA K); 16.III.1995 (fl) Costa, M. A. S. et al. 164 (NOK); 28.IV.1988 (bd) Ramos, J. F. \& Lima, R. P. 1887 (INPA K MG NY SP); 2.VI.1993 (fl) Ribeiro, J.E. L. S. et al. 786 (INPA); 3.VI.1993 (fl) Ribeiro, J. E. L. S. et al. 821 (INPA K); 26.IV.1994 (fr) Vicentini, A. et al. 490 (INPA K MG MO NY RB SP). 
Hypolytrum schraderianum is distinguished by its paniculate inflorescence, two floral bracts and two stamens per spicoid.

\section{Kyllinga}

Annual or perennial herbs. Rhizome short or horizontally creeping. Leaves basal, 3-ranked, the blades elongated or reduced; ligule 0 . Involucral bracts leaf-like. Inflorescence capitate. Spikes 1-few, sessile, cylindric, ellipsoid or globose; axis short. Spikelets numerous, falling entire, crowded, bilaterally flattened. Glumes several, \pm equal in length, 2-ranked, strongly laterally flattened, sides membranous to hyaline, nerves 0 several, keel strongly acute, sometimes winged, smooth, spinulose or serrulate. Flowers 1-5 per spikelet, bisexual or staminate in upper glume(s). Perianth segments 0. Stamens 2-3. Stigmas 2; style continuous with ovary. Nutlets $1-2$ per spikelet, laterally biconvex with one margin facing the axis.

Genus of $c a .60$ species in tropical, subtropical and warm-temperate regions with the highest species diversity in Africa.

\section{Key to the species of Kyllinga in Reserva Ducke}

1. Perennial; rhizome long-creeping; spikes 1 per inflorescence 1. K. brevifolia

1. Annual; rhizome absent; spikes usually 2-3 per inflorescence 2. K. pumila

9.1 Kyllinga brevifolia Rottb., Descr. Icon. Rar. Nov. Pl. 13, t. 4, fig. 3. 1773; Nees in Mart., Fl. bras. 2(1): 15. 1842.

Cyperus brevifolius (Rottb.) Hassk., Cat. Hort. Bogor. 24. 1884; Kük. in Engl., Pflanzenr. 4(20), 101 Heft: 600. 1936.

Perennial. Rhizome long-creeping. Culms rather distant in series along rhizome, $7-30 \mathrm{~cm}$ long, $1-1.5 \mathrm{~mm}$ wide, triquetrous, smooth. Leaves: blade narrowly linear, 2$17 \mathrm{~cm}$ long, 1-3 mm wide, acute, flattishplicate; sheath 1-20 cm long, brownish or purplish brown. Involucral bracts $2-4$, the longest 3-20 cm long, 1.7-2.4 mm wide, sometimes erect. Inflorescence capitate, globose. Spikes 1(-3), globose, $0.5-1 \times 0.5-$ $1 \mathrm{~cm}$. Spikelets oblong-lanceolate to ellipticlanceolate, 3-3.5 × $1 \mathrm{~mm}, 1(-2)$-flowered. Glumes ovate-elliptic, 1-3.5 mm long, shortly cuspidate, sides membranous, 5-7-nerved, pale green to pale brown, keel sparsely spinulose, green. Stamens 1-2(-3); anthers $1 \mathrm{~mm}$ long. Nutlets $1-2$ per spikelet, obovate or elliptic, $1-1.5 \times 0.5-0.7 \mathrm{~mm}$, brownish, minutely punctate.

Tropics, subtropics and warm temperate regions.

Open damp or wet places.
8.VI.1995 (fl) Costa, M. A. S. \& Silva, C. F. 303 (INPA K MG MO NY R RB SP UL); 26.IV.1981 (fl) Lowe, J. 4099 (INPA K UIH); 14.XII.1966 (fl) Prance, G. T. et al. 3638 (INPA).

Kyllinga brevifolia is a common pantropical weed and is recognised by its creeping rhizome with culms that are rather distant along the rhizome.

9.2 Kyllinga pumila Michx, Fl. bor.-amer. 1: 28. 1803; Nees in Mart., Fl. bras. 2(1): 20. 1842 .

Cyperus densicaespitosus Mattf. \& Kük. in Engl., Pflanzenr. 4(20), 101 Heft: 597. 1936.

Annual. Rhizome absent. Culms 14$30 \mathrm{~cm}$ long, $0.4-0.6 \mathrm{~mm}$ wide, trigonous to \pm terete, smooth. Leaves: blade narrowly linear, 3-9 cm long, 0.7-1.7 mm wide, flattish to boated-shaped in cross section; sheath 2.5$5 \mathrm{~mm}$ long, dark reddish-brown. Involucral bracts 4 , the longest $5-7 \mathrm{~cm}$ long, $0.6-1 \mathrm{~mm}$ wide. Inflorescence capitate, globose. Spikes usually 3 , broadly ovoid to subglobose, $5 \times$ $4 \mathrm{~mm}$; spikelets lanceolate, $2 \times 0.5 \mathrm{~mm}, 1$ flowered. Glumes ovate-lanceolate, 1.5-2 $\times$ $1 \mathrm{~mm}$, shortly cuspidate, sides membranous, sides 3-4-nerved, pale green, keel somewhat winged spinulose. Stamen 1; anthers $0.3 \mathrm{~mm}$ 
long. Nutlets 1 per spikelet, elliptic-obovate, $1 \times 0.5 \mathrm{~mm}$, greenish-brown, minutely puncticulate.

Tropical America and Africa.

8.VI.1995 (fr) Costa, M. A. S. \& Silva, C. F. 305 (G INPA K MG MO NY R RB SP U).

Open, damp places.

Kyllinga pumila is an annual species without a rhizome and often has three spikes per inflorescence.

\section{Mapania}

Stoloniferous or rhizomatous perennial herbs. Rhizome woody. Culms central or lateral (arising from lower leaf axils or below the leaves), the latter with cataphylls at base. Leaves 3-ranked, basal or cauline; blade linear to oblong, coriacous; pseudopetiole present or 0 ; ligule 0 . Involucral bracts leaflike in capitate and centrally culmed species, otherwise glume-like. Inflorescence paniculate, capitate with few to many spikes (in Ducke taxa) or a single spike only. Spikes comprising few-many spirally imbricate glume-like bracts (spicoid bracts), each subtending a partial inflorescence with a much reduced axis (spicoid). Spicoid comprising a naked, terminal female flower and 4-6 scalelike floral bracts, the lowest 2 opposite, keeled, the lowest 3 subtending a male flower the remainder empty. Perianth segments 0 . Stamens 1-3 per spicoid, 1 per male flower. Stigmas 2-3. Nutlets with a hard or succulent exocarp, smooth or sculptured, lateral costae or furrows 0 or $2-3$.

Genus of 80 species, Sri Lanka and N. India, S. China, Indo-China, Malesia, northeastern Australia and Polynesia; also in tropical Africa, S. Central America and N. South America.

\section{Key to the species of Mapania in Reserva Ducke}

1. Leaves reduced to bladeless sheaths; involucral bracts elliptic to elliptic-obovate

3. M. sylvatica

1. Leaves with a linear blade; involucral bracts linear.

2. Leaves more than $2 \mathrm{~cm}$ wide; inflorescence reddish-brown; nutlet without spongy apex and dark brown spot towards base 1. M. pycnocephala

2. Leaves up to $2 \mathrm{~cm}$ wide; inflorescence whitish; nutlet with spongy apex and dark brown spot towards base

2. M. pycnostachya

10.1 Mapania pycnocephala subsp. fluviatilis (Sandwith) T. Koyama, Mem. N.Y. Bot. Gard. 17: 66. 1967; D. A. Simpson, Rev. gen. Mapania: 152. 1992.

Mapania fluviatilis Sandwith, Kew Bull. 1933:495. 1933.

Perennial. Rhizome short. Culm central, $35-70 \mathrm{~cm}$ long, $1.9-4.5 \mathrm{~mm}$ wide, subtriquetrous, glabrous. Leaves basal; blade linear, rarely linear-oblong, $27-112 \mathrm{~cm}$ long, $2.1-7.6 \mathrm{~cm}$ wide, narrowed, acuminate, base narrowed into pseudopetiole, $1(-3)$-nerved; pseudopetiole 5-22 cm long; sheath 12-18 cm long, dark reddish-brown. Involucral bracts 3-4, leaf-like, linear, 8-89 ×0.2-7.7 cm, basal bract longest. Inflorescence capitate, halfglobose to globose, $2-6.5 \mathrm{~cm}$ wide, mid- reddish-brown, with numerous spikes. Spikes linear to elliptic, up to $1.5 \mathrm{~cm}$ long, often indistinct. Spicoid bracts linear-lanceolate or lanceolate, 6.5-7.7 × 1.4-2.8 mm, acute, mid-reddish-brown. Spicoids shorter or \pm equalling spicoid bracts. Floral bracts 4 , free to \pm connate, lower 2 bracts linear, $6.2-7.3 \times$ $0.6-1.2 \mathrm{~mm}$, keel usually narrowly winged, glabrous to sparsely hispid. Stamens 2 per spicoid, anthers linear, linear-oblong, 2.3$3 \mathrm{~mm}$ long; stigma branches 2 . Nutlets obovoid to globose, $1-1.5 \times 0.9-1.4 \mathrm{~mm}$, rounded, apex apiculate, not spongy; surface smooth, light greenish or brown at first, becoming uniformly dark brown and without a dark brown spot towards base, often shiny, lateral costae 2 , indistinct. 
South America: Venezuela, Guyana, French Guiana and Brazil.

Forest.

30.VII.1996 (fl) Luceño, M. \& A. P. Mendes 01 (INPA).

Additional specimens examined: BRAZIL. AMAPÁ: Río Amapari, Serra do Navio, along trail to Río Araguary, 19.XI.1954 Cowan 38439 (NY); Río Vila Nova, 8.XII.1976 Ribeiro 1659(NY UPS); Tumucumaque, $2.5 \mathrm{~km}$ NNW of Mitaraka, 1.IX.1972 de Granville 1449 (CAY NY); AMAZONAS: Río Negro, São Gabriel de Cachoeira, 21.X.1978 Madison et al. 6534 (AAU K NY); 40 km from São Gabriel 21.X.1978 Nascimento 712 (NY UPS).

Mapania pycnocephala subsp. fluviatilis is distinguished by its reddish-brown inflorescence with numerous spikes and two stamens per spicoid. Material from Costa Rica to western Venezuela is assigned to subsp. pycnocephala which has shorter spicoid bracts and a nutlet surface that is shallowly rugulose.

10.2 Mapania pycnostachya (Benth.) T. Koyama, Mem. N.Y. Bot. Gard. 17: 61. 1967; D. A. Simpson, Rev. gen. Mapania: 165. 1992.

Diplasia pycnostachya Benth., J. Linn Soc. 15: 512. 1877.

Hypolytrum condensatum C.B. Clarke, Kew Bull. Add. Ser. 8: 50. 1908, nom. superfl.

Perennial. Rhizome short. Culm central, 35-50 cm long, 2.2-2.9 mm wide, trigonous, scabrid. Leaves basal; blade linear, 49-82 $\times$ $1.4-2 \mathrm{~cm}$, gradually narrowed, acuminate, base gradually narrowed into pseudopetiole, 1-nerved; pseudopetiole 2.5-8 × 0.4-6 cm; sheath $5-7 \mathrm{~cm}$ long, greenish to reddishbrown. Involucral bracts 3(-4), leaf-like, linear, 5-67 ×0.2-2 cm, basal bract longest. Inflorescence capitate, globose, $1.5-2.5 \mathrm{~cm}$ in diam., whitish, with up to 20 spikes. Spikes ovate, $1 \times 0.5 \mathrm{~cm}$, usually indistinct. Spicoid bracts lanceolate, $6.8-8 \mathrm{~cm} \times 1.9-2.7 \mathrm{~mm}$, acute, light brown. Spicoids \pm equalling spicoid bracts. Floral bracts 4 , free, lower 2 bracts lanceolate, $6.8-8 \times 0.9-1.1 \mathrm{~mm}$, keel glabrous. Stamen 1 per spicoid, anther linearoblong, 2-2.3 mm long, cream; stigma branches 2. Nutlets 2-sided, ovoid or ellipsoid,
3.5-4.5 × 1.5-1.9 mm, apex spongy, acute; surface smooth or slightly wrinkled, shiny, light brown, with a dark brown spot towards base, lateral costae absent.

South America: Southern Venezuela and northern Brazil.

Margins of streams in forest.

14.IX.1971 (fr) Prance, G. T. et al. 14744 (F INPA K NYU US).

Mapania pycnostachya has a whitish inflorescence, one stamen per spicoid and a nutlet with a spongy section at the apex and distinct brown spot towards the base.

10.3 Mapania sylvatica Aubl. subsp. sylvatica, Hist. Pl. Guiane Fr. 1: 47; 3: t.17. 1775; D. A. Simpson, Rev. gen. Mapania: 43. 1992.

Perennial. Rhizome short. Culm central, 21-60 cm long, 1.4-2.9 mm wide, terete to subtrigonous, glabrous. Leaves reduced to bladeless sheaths; sheaths 2.5$18 \mathrm{~cm}$ long, dark reddish. Involucral bracts $3(-4)$, leaf-like, narrowly elliptic or ellipticobovate, $11-30 \times 3.2-6 \mathrm{~cm}, \pm$ equal in size. Inflorescence terminal, with 1-2, rarely more spikes. Spikes elliptic to oblong, rarely oblong-lanceolate, $1.2-2.7 \times 0.8-1.4 \mathrm{~cm}$, distinct. Spicoid bracts narrowly oblong, $5.5-$ $6.5 \times 1.4-2.2 \mathrm{~mm}$, acute, often somewhat cucullate, often splitting longitudinally, reddish-brown. Spicoids \pm equalling or exceeding spicoid bracts. Floral bracts 6 , free, lowest 2 bracts linear or linearlanceolate, $6.3-7.9 \times 1.2-1.5 \mathrm{~mm}$, acute, sometimes cucullate, reddish-brown, keel sparsely to densely ciliate. Stamens 3 per spicoid, anthers linear, linear-oblong, 1.3$2.4 \mathrm{~mm}$ long; stigma branches 3. Nutlets obovoid to subglobose, $1.5-2.5 \times 1.1-$ $1.5 \mathrm{~mm}$, rounded, shortly apiculate; surface strongly longitudinally ridged, with connecting horizontal ridges, green at first, becoming dull olive brown or dark brown, lateral costae 3 .

Northern South America.

Wet forest, often near to water. 19.I.1995 (fl) Costa, M. A. S. \& Nascimento, J. R. 111 (INPA K MG MO NY SP); 4.VIII.1994 (fr) 
Ribeiro, J. E. L. S. \& Silva, C. F. 1389 (INPA K MG, MO NY RB SP); 1.XII.1956 (fl) Rodrigues, W. \& Chagas, J. 1470 (INPA); 8.X.1963 (fl) Rodrigues, W. 5493 (INPA).

Mapania sylvatica subsp. sylvatica is an unusual species with leaves reduced to bladeless sheaths and elliptic to elliptic-obovate involucral bracts. The inflorescence usually has 1-2 spikes, there are six floral bracts per spicoid and the nutlet is obovoid to subglobose.

\section{Pleurostachys}

Perennial herbs. Rhizome shortcreeping. Culms noded. Leaves mostly cauline, a few basal and often reduced to a bladeless sheath. Involucral bracts usually leaf-like. Inflorescence paniculate; partial inflorescences corymbose to umbel-like, rarely contracted. Spikelets small, usually terete. Glumes several to many, spirally imbricate or rarely distichous, the basal glumes small, empty, the remainder gradually inceasing in length towards the spikelet apex, with the middle 3-7 glumes subtending a flower and the upper few empty. Flowers bisxual. Perianth segments 3-6, upper part ciliate to fimbriate, lower part scabrid. Stamens 3. Stigmas 2. Nutlets biconvex, often rugose; style base persistent often thickened.

Genus of $c a .30$ species, subtropical and tropical S. America.

11.1 Pleurostachys sparsiflora Kunth, Enum. Pl. 2: 286. 1837.

Nemochloa sparsiflora (Kunth) Nees in Mart., Fl. bras. 2(1): 151. 1842.

Perennial. Rhizome woody. Culms somewhat distant, usually hidden by cauline leaf sheaths, $17-37 \mathrm{~cm}$ long, $1.5-2.5 \mathrm{~mm}$ wide, trigonous, smooth. Leaves: blade linear, $27-35 \mathrm{~cm}$ long, 4-6 mm wide, gradually narrowed, acuminate, flat, green; sheath 3$3.5 \mathrm{~cm}$ long, green to pale brown. Involucral bracts leaf-like up $30 \mathrm{~cm}$ long. Inflorescence paniculate; nodes 6-8, distant, each subtending 1-2 partial inflorescences; partial inflorescences corymbose, simple to compound, 10-19 cm long; primary branches filamentous, 4-8 cm long; secondary branches $1-2.5 \mathrm{~cm}$ long. Spikelets 1-2 per inflorescence branch, obovoid, $2-3.5 \times 1.3-$ $2 \mathrm{~mm}$, obtuse, mid-brown. Glumes ovate, $2 \mathrm{~mm}$ long, $1 \mathrm{~mm}$ wide, obtuse, membranous, mid-reddish-brown. Nutlets broadly ovate, $2.8-3 \times 1.6-1.8 \mathrm{~mm}$, rounded, mid-brown, surface irregular, style base conical, blackish.

Tropical South America.

Forest.

17.I.1995 (fl) Costa, M. A. S. \& Nascimento, J. R. 103 (INPA K MG MO NY R RB SP U); 17.V.1988 (fr) Coêlho, D. \& Lima, R. P. 52-D(INPA K MG MO NY RB SP); 7.VIII.1995 (st) Nee, M. 46211 (K).

Additional specimen examined: BRAZIL. PARÁ: Itatuba 17.XI.1978 Silva et al. 3799 (K).

Pleurostachys sparsiflora is recognised by its corymbose partial inflorescences that have filamentous branches and small spikelets borne singly at the tips of the branches.

\section{Rhynchospora}

Annual or perennial herbs. Culms central. Leaves basal and/or cauline; blades linear to lanceolate; sheaths closed; ligule 0 . Involucral bracts usually leaf-like. Inflorescence capitate or paniculate; partial inflorescences umbel-like or corymbose. Spikelets lanceolate, ovate to elliptic, flattened to terete. Glumes 5-9 (rarely more), 2-ranked or spirally imbricate, membranous to chartaceous, 1 nerved, the basal 2-3 glumes, empty and small, the remainder gradually inceasing in length towards the spikelet apex, with a single flower, the uppermost glume often empty. Flowers either all bisexual, the upper ones not maturing a nutlet, or lower 1 -few bisexual and upper ones male, or unisexual with the lowest female and upper one(s) male. Perianth segments $0-6$, rarely more, bristle-like, upwardly or retrorsely scabrid, rarely smooth. Stamens (1-)2-3. Stigmas 2 or style undivided. Nutlets biconvex, smooth, cancellate, rugose or sometimes spinose; style base persistent, variously-shaped. 
Over 250 species in temperate and tropical regions, with the greatest concentration of species in tropical and subtropical South America.

12.1 Rhynchospora pubera (Vahl) Boeck subsp. pubera, Linnaea 37: 528. 1872,.

Dichromena pubera Vahl subsp. pubera, Enum. Pl. 2: 241. 1806.

Perennial. Culms tufted, 7-28 cm long, 0.4-0.7 $\mathrm{mm}$ wide, terete to \pm trigonous, smooth. Leaves basal; blade linear, up to $19 \mathrm{~cm}$ long, 1-2.5 mm wide, gradually narrowed, subacute, flattish to folded; sheaths up to $1-3 \mathrm{~cm}$ long, pale brown. Involucral bracts 4, leaf-like, greenish to whitish at base, the longest up to $8 \mathrm{~cm}$ long. Inflorescence capitate, ovoid to subglobose, $0.7-0.9 \times 0.7-$ $1 \mathrm{~cm}$. Spikelets $2-6$, lanceolate, \pm terete, $0.7-$ $0.8 \mathrm{~cm} \times 2-3 \mathrm{~mm}$, white. Glumes 8-10, ovate to lanceolate, $5.5-6 \times 2.2-2.4 \mathrm{~mm}$, white, keel often ciliolate. Perianth segments 0 . Stamens 3; anthers $3 \mathrm{~mm}$ long. Stigmas 2. Nutlets lenticular, widely obovate, $2.5 \mathrm{~mm}$ long, $1.2 \mathrm{~mm}$ wide, mid- to very dark brown, transversely rugose; style-base very shallowly triangular.

Northern South America, mostly E. of Colombia and Peru.

Open, damp places.

8.VI.1995 (fl) Costa, M. A. S. \& Silva, C. F. 304 (INPA KMGMONY RB SPU); 26.IV.1981 (fl) Lowe, J. 4101 (INPA).

Rhynchospora pubera subsp. pubera is distinguished by its white spikelets and the lenticular nutlets with a shallowly triangular style-base and transversely rugose surface. It is one of a number of species in the genus Rhynchospora that are insect-pollinated.

\section{Scleria}

Annual or perennial herbs. Rhizome usually woody, often knotted. Culms solitary or tufted, usually erect, sometimes climbing or scrambling. Leaves basal and/or cauline, the latter sometimes apparently in whorls; blade mostly linear; sheath closed, often 3winged; ligule 0 ; contraligule usually present. Inflorescence usually paniculate, bearing a terminal and 0 -several lateral partial inflorescences, occasionally reduced and spike-like or capitate. Spikelets unisexual or bisexual; bisexual spikelets with terminal female flower and 1-several lateral male ones; female spikelets with a single female flower and 1-several lateral glumes (reduced male flowers); male spikelets with several to many glumes. Glumes spirally arranged or 2ranked. Flowers unisexual. Perianth segments 0. Stamens 1-3 per male flower. Stigmas 3; style continuous with the ovary, deciduous. Nutlets not spongy, not enclosed by a membranous sac, terete or subtrigonous, mostly globose, ovoid or subpyramidal, with bony pericarp and a stipe-like, 3-lobed or cup-like, rarely indistinct disk attached at the base.

\section{Key to the species of Scleria in Reserva Ducke}

1. Plants climbing or trailing; disk at nutlet base an irregular ring... 3. S. secans

1. Plants erect, not climbing; disk at nutlet base 3-lobed, the lobes rounded or fimbriate.

2. Inflorescence spike-like; leaf blade $15 \mathrm{~mm}$ or more wide; disk-lobes fimbriate... 1. S. cyperina

2. Inflorescence elongated; leaf blade up to $5 \mathrm{~mm}$ wide; disk-lobes rounded ..2. S. melaleuca

13.1 Scleria cyperina Kunth, Enum. Pl. 2: 345. 1837.

Erect perennial. Rhizome creeping. Culms to $37 \mathrm{~cm}$ long, $1.5-2.5 \mathrm{~mm}$ wide, triquetrous, glabrous. Leaves cauline; blade linear, to $52 \mathrm{~cm}$ long, $15-17 \mathrm{~mm}$ wide, gradually narrowed, narrowly obtuse, flattish; sheath 7$9 \mathrm{~cm}$ long, pale brown to reddish-tinged; contraligule broadly obtuse, glabrous often with a broad, membranous apex. Lowest involucral bract leaf-like, up to $26 \mathrm{~cm}$ long; upper bracts indistinct, setaceous. 
Inflorescence paniculate, but appearing spikelike, $8-10 \times 4-8 \mathrm{~cm}$; nodes $12-22$, crowded or the lowermost one distant, each subtending a single partial inflorescence or the upper ones a single male spikelet. Spikelets female and male, solitary; female spikelets sessile, obovoid, 5-6 mm long; male spikelets pedunculate, oblong-lanceolate, 4-5 $\mathrm{mm}$ long, peduncle up $1 \mathrm{~mm}$ long. Female glumes elliptic-ovate, 5$7 \times 2 \mathrm{~mm}$, acute, sides coriaceous, very dark reddish, keel slightly paler or greenish. Stamen 1. Nutlets obovoid-globose, terete-trigonous, $3.2 \times 2 \mathrm{~mm}$, rounded, dark purplish above white below, indistinctly reticulate, rather shiny, sparsely pubescent below; disk 3-lobed, the lobes fimbriate.

Tropical South America.

Open areas.

20.I.1995 (fr) Costa, M. A. S. \& Nascimento, J. R. 113 (INPA K MG MO NY RB SP).

Additional specimen examined: BRAZIL. AMAZONAS: Manaus-Pôrto Velho highway, km 245 13.III.1974 Prance et al. 20463 (INPA NY K).

Scleria cyperina has rather broad leaves and a crowded spike-like inflorescence. The disk-lobes at the nutlet base are fimbriate.

13.2 Scleria melaleuca $\mathrm{Rchb}$. ex Schltdl. \& Cham., Linnaea 6: 29. 1831; Nees in Mart., Fl. bras. 2(1): 178. 1842.

Scleria pratensis Lindl. ex Nees, in Mart., Fl. bras. 2,1: 179. 1842.

Erect perennial. Rhizome shortcreeping. Culms to $19 \mathrm{~cm}$ long, $1 \mathrm{~mm}$ wide, subtriquetrous, glabrous to puberulent. Leaves mostly cauline; blade linear, $15-28 \mathrm{~cm}$ long, $5 \mathrm{~mm}$ wide, gradually narrowed, obtuse, flat to plicate; sheath 6-9 $\mathrm{cm}$ long, pale brown to reddish; contraligule shallowly rounded, pubescent. Lowest involucral bract leaf-like, up to $26 \mathrm{~cm}$ long, upper bracts rather indistinct, setaceous. Inflorescence elongated, narrowly paniculate, open, $14-26 \times 2 \mathrm{~cm}$; nodes 3-4, each subtending a single partial inflorescence; partial inflorescence sessile or shortly pedunculate, ovate-lanceolate, $2-8 \mathrm{~cm}$ long, decreasing in size towards apex. Spikelets female and male, solitary or in groups of 2-3; female spikelets sessile, obovoid, 3-3.5 mm long; male spikelets shortly pedunculate, oblong-lanceolate, $3.5 \mathrm{~mm}$ long. Female glumes elliptic-ovate, $3 \times 2 \mathrm{~mm}$, acute, sides coriaceous, dark reddish-brown, keel green. Stamen 1. Nutlets globose, terete, $2.5 \times 2 \mathrm{~mm}$, rounded, dark purplish above white below, smooth, shiny, sparsely pubescent below; disk 3-lobed, the lobes rounded.

Subtropical and tropical America, tropical Africa, Madagascar.

Open, damp areas.

8.VI.1995 (fr) Costa, M. A. S. \& Silva, C. F. 306

(INPA K MG MO NY R RB SPU).

Scleria melaleuca has narrow leaves and an elongated inflorescence. The disk-lobes are rounded.

13.3 Scleria secans (L.) Urb., Symb. Ant. 2(1): 169. 1900.

Schoenus secans L., Syst. Nat. 10,2: 1759.

Climbing or trailing perennial. Rhizome creeping, knotted. Culms up to $10 \mathrm{~m}$ long, 1.5$2.5 \mathrm{~mm}$ wide, triquetrous, glabrous to scabrid. Leaves cauline; blade linear, $28 \mathrm{~cm}$ long, 4$5 \mathrm{~mm}$ wide, gradually narrowed, acuminate, flat to plicate, margins sharply minutely toothed; sheath $3-5.5 \mathrm{~cm}$ long, pale to mid-brown, pubescent above; contraligule obtuse, pubescent. Lowest involucral bract leaf-like, up to $21 \mathrm{~cm}$ long, upper bracts leaf-like to setaceous. Inflorescence elongated, narrowly paniculate, open, 13-14 × 1-2 cm; nodes 79 , each subtending a single partial inflorescence; partial inflorescence sessile to pedunculate, $0.5-5 \mathrm{~cm}$ long. Spikelets female and male, solitary or sometimes male spikelets in groups of 2; female spikelets sessile, obovoid, 5-6 mm long; male spikelets shortly pedunculate, narrowly-lanceolate, $4 \mathrm{~mm}$ long. Female glumes broadly elliptic, $5 \times 3 \mathrm{~mm}$, acute, sides coriaceous, green or brown with dark reddish-brown margins, keel green. Stamen 1. Nutlets ovoid, terete-trigonous, $2.8 \times 2.5 \mathrm{~mm}$, rounded, white, smooth, shiny; disk forming an irregular ring.

Tropical America.

Open areas. 
10.V.1988 (fr) Coêlho, D. \& Lima, R. P. 34-D (INPA K). Additional specimens examined: BRAZIL. AMAZONAS: $50 \mathrm{~km}$ NE of Manaus, 1.V.1981 Lowe 4113 (INPA K); MINAS GERAIS: Serra do Espinhaço, Lapinha, 24.II.1968 Irwin et al. 20768 (K NY).
Scleria secans is one of the few climbing species of Cyperaceae and may ascend up to $10 \mathrm{~m}$. The minutely toothed leaf-margins easily lacerate the skin. The disk forms an irregular ring around the nutlet base. 